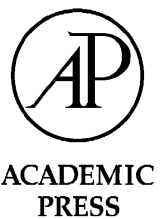

Available online at www.sciencedirect.com

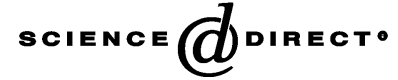

Journal of Sound and Vibration 271 (2004) 257-277

\title{
Dynamic analysis of submerged fluid-filled pipelines subjected to a point pressure load
}

\author{
L. Godinho*, A. Tadeu, F.J.G. Branco \\ Department of Civil Engineering, University of Coimbra, Polo II - Pinhal de Marrocos, 3030-290 Coimbra, Portugal
}

Received 10 May 2002; accepted 24 February 2003

\begin{abstract}
This paper analyses the wave scattering generated by point pressure loads in the vicinity of infinite fluidfilled circular pipelines submerged in a homogeneous fluid medium. The pipeline has a constant crosssection and is modelled as a homogeneous elastic material. The three-dimensional (3-D) response is formulated in the frequency domain, and is obtained as a discrete summation of the 2-D solutions found for different axial wavenumbers. Time solutions are computed by means of inverse Fourier transforms. Complex frequencies are used to avoid aliasing phenomena.

The main focus of the paper is on the dynamic analysis of the stresses generated inside and at the surface of the pipeline by a point pressure load placed in the surrounding medium. Different positions of both the source and the receivers are considered. The effect of the relation of the wall thickness to the radius of the pipeline is also studied.
\end{abstract}

(C) 2003 Elsevier Ltd. All rights reserved.

\section{Introduction}

The vibration of shell and tubular structures has been studied using different approaches in order to identify features of wave propagation and to detect and identify submerged structures, the presence of cracks and structural imperfections. Analytical methods are applicable only to simpler geometries, while more general numerical methods, such as the finite element method and the boundary element method, allow the study of a wider range of geometric configurations.

Liu and $\mathrm{Qu}$ [1] provided some guidelines on using guided waves to detect radial cracks in annular structural components, such as those found in ageing helicopters. The inner surface of the annular structure is assumed to be traction free while the outer surface is subject to a

\footnotetext{
*Corresponding author. Fax: +351-239-797-190.

E-mail address: lgodinho@dec.uc.pt (L. Godinho).
} 
time-dependent transient excitation. They used the method of eigenfunction expansion to analyze the propagation of transient waves generated at transducers with different incidence angles in a circular annulus. With this method, the contributions of the different eigenmodes are separated, allowing the ones making the more important contributions to the response to be identified.

Chung and Lee [2] analyzed how the behaviour of axisymmetric shell structures changes when small imperfections are introduced. They proposed the use of a new conical ring element to be used in connection with the finite element method. This element accounted for the possible effects of slight local deviations from the pure axisymmetric form. This method was applied to the vibration analysis of a bell.

The study of wave propagation in circular cylindrical shells that are either filled with, or submerged in, a fluid requires the use of models that take into account the coupling between the solid and the fluid media.

Different models have been proposed by many researchers to solve this type of problem. Veksler et al. [3] studied the features of wave scattering by submerged elastic circular cylindrical shells, filled with air, when struck by plane harmonic acoustic waves. Their work used the standard resonance scattering theory to study the modal resonances. Their investigation was focused on the generation of bending waves, presenting a set of dispersion curves for shells with different relative thicknesses. They concluded that these waves could be generated when the relative thickness of the shell is not too great, and that the dispersion curves of their phase velocity are limited by the dispersion curve of the free bending modes, when the density of the host fluid tends to zero.

Work by Bao et al. [4] analyzed the existence of various types of circumferential waves and the repulsion of their dispersion curves for the case of a thin elastic circular cylindrical shell submerged in one fluid and filled with another fluid. Their study was based on an analytical calculation of the partial-wave resonances in the acoustic scattering amplitude of a normally incident plane wave.

Recently, Maze et al. [5] studied the various guided acoustic circumferential modes found in a water-filled tube. They showed, theoretically and experimentally, the presence of structure waves inside a water-filled thin walled tube in vacuum. The values of the group velocities for the first two coupled modes calculated by the authors were in excellent agreement with experimental results. The results were interesting in the context of the physics of acoustic waves in fluid loaded structures and may be applied in liquid level detection.

This paper examines the three-dimensional wave propagation in a fluid-filled pipeline solid structure, submerged in a continuous homogeneous fluid medium. The full coupling between the external fluid, the elastic material and the internal fluid is taken into account. The pipeline is assumed to have a constant cross-section and is modelled as a homogeneous elastic material.

Since the geometry of the problem does not change along the axis of the pipeline $(z)$ and to avoid the full three-dimensional computation, the problem is first Fourier transformed along this axis, which allows the solution to be defined in a frequency vs. axial wavenumber domain. An inverse Fourier transform is subsequently performed along the same direction to find the threedimensional solution in the frequency domain. Assuming the existence of a set of virtual sources, equally spaced along the $z$ direction, the resulting Fourier transform becomes discrete. This procedure allows the authors to obtain the three-dimensional (3-D) responses in the frequency domain as a discrete summation of the 2-D solutions for different axial wavenumbers [6]. Each of 
the 2-D problems is solved analytically. This model is then employed to compute stresses in the elastic material of a submerged fluid-filled pipeline, and to analyze their dependence on the excitation of the different normal modes. To better understand the behaviour of the dynamic system, time domain responses are also obtained by applying inverse Fourier transforms in the frequency domain. The wave propagation features identified in the present paper should be useful in the development of non-destructive testing and imaging methods.

The next section of this work describes briefly the formulation of the three-dimensional problem. A brief description of the analytical solutions used to calculate the frequency domain responses is then presented, followed by an explanation of the process used to compute time domain results. Finally, a set of numerical applications is presented to show the behaviour of an infinite pipeline subjected to the effect of a point source placed in different positions. The response is calculated at receivers placed in the solid material of the pipeline, and for pipelines with different thicknesses.

\section{3-D problem formulation}

In the absence of body forces, and disregarding the effects of the mean fluid flow velocity, the displacement equation of motion in an infinite 3-D elastic homogeneous medium can be written, in the frequency domain $[7,8]$, as

$$
(\lambda+2 \mu) \boldsymbol{\nabla}(\boldsymbol{\nabla} \cdot \mathbf{u})-\mu \boldsymbol{\nabla} \times(\boldsymbol{\nabla} \times \mathbf{u})=-\omega^{2} \rho \mathbf{u},
$$

where $\mathbf{u}$ is the displacement vector, $\mu$ is the shear modulus, $\lambda$ the Lamé constant, $\rho$ the density of the medium and $\omega$ the frequency of excitation.

In order to solve this elastic equation, one can separate the displacement vector into different components, corresponding to one dilatational $(\phi)$ and two shear $(\psi$ and $\chi)$ (vertically polarized, $\mathrm{SV}$ and horizontally polarized, $\mathrm{SH}$ ) components, which satisfy the scalar equations

$$
\begin{gathered}
\left(\nabla^{2}+\frac{\omega^{2}}{\alpha^{2}}\right) \phi(\omega, r, \theta, z)=0 \\
\left(\nabla^{2}+\frac{\omega^{2}}{\beta^{2}}\right) \psi(\omega, r, \theta, z)=0 \\
\left(\nabla^{2}+\frac{\omega^{2}}{\beta^{2}}\right) \chi(\omega, r, \theta, z)=0
\end{gathered}
$$

with

$$
\boldsymbol{\nabla}^{2}=\left(\frac{\partial^{2}}{\partial r^{2}}+\frac{1}{r} \frac{\partial}{\partial r}+\frac{1}{r^{2}} \frac{\partial^{2}}{\partial \theta^{2}}+\frac{\partial^{2}}{\partial z^{2}}\right), \quad r=\sqrt{x^{2}+y^{2}}, \quad \alpha^{2}=(\lambda+2 \mu) / \rho, \quad \beta^{2}=\mu / \rho,
$$

$\alpha$ and $\beta$ being the dilatational and the shear wave velocities respectively. 
In the case of an inviscid compressible fluid medium, no shear stresses are allowed, and so only dilatational waves can exist. For this case, the system is reduced to a single scalar equation

$$
\left(\nabla^{2}+\frac{\omega^{2}}{\alpha_{f}^{2}}\right) \phi_{f}(\omega, r, \theta, z)=0,
$$

where $\phi_{f}$ is the velocity potential and $\alpha_{f}$ is the wave propagation velocity in the fluid.

In many cases, the analysis of 3-D problems can be computationally demanding, and it is often advisable to express the full 3-D problem as a summation of simpler 2-D solutions, when the geometry of the problem remains constant along one direction $(z)$. This is achieved by applying a Fourier transformation along that direction, and expressing the solution as a summation of 2-D solutions, with different spatial wavenumbers $k_{z}$ [9]. The application of a spatial Fourier transformation along the $z$ direction to Eqs. (2) and (3) leads to the following equations:

$$
\begin{aligned}
& {\left[\hat{\boldsymbol{\nabla}}^{2}+\left(\frac{\omega^{2}}{\alpha^{2}}-k_{z}^{2}\right)\right] \hat{\phi}\left(\omega, r, \theta, k_{z}\right)=0,} \\
& {\left[\hat{\boldsymbol{\nabla}}^{2}+\left(\frac{\omega^{2}}{\beta^{2}}-k_{z}^{2}\right)\right] \hat{\psi}\left(\omega, r, \theta, k_{z}\right)=0,} \\
& {\left[\hat{\boldsymbol{\nabla}}^{2}+\left(\frac{\omega^{2}}{\beta^{2}}-k_{z}^{2}\right)\right] \hat{\chi}\left(\omega, r, \theta, k_{z}\right)=0,} \\
& {\left[\hat{\boldsymbol{\nabla}}^{2}+\left(\frac{\omega^{2}}{\alpha_{f}^{2}}-k_{z}^{2}\right)\right] \hat{\phi}_{f}\left(\omega, r, \theta, k_{z}\right)=0}
\end{aligned}
$$

with

$$
\hat{\boldsymbol{\nabla}}^{2}=\left(\frac{\partial^{2}}{\partial r^{2}}+\frac{1}{r} \frac{\partial}{\partial r}+\frac{1}{r^{2}} \frac{\partial^{2}}{\partial \theta^{2}}\right) .
$$

The full three-dimensional solution is then obtained by applying an inverse Fourier transform along the $z$ direction. If we assume the existence of virtual sources spaced at equal intervals, $L$, along $z$, this inverse Fourier transformation becomes a discrete summation, which allows the solution to be obtained by solving a limited number of two-dimensional problems.

$$
\begin{aligned}
& \phi(\omega, r, \theta, z)=\frac{2 \pi}{L} \sum_{m=-M}^{M} \hat{\phi}\left(\omega, r, \theta, k_{z m}\right) \mathrm{e}^{-\mathrm{i} k_{z m} z}, \\
& \psi(\omega, r, \theta, z)=\frac{2 \pi}{L} \sum_{m=-M}^{M} \hat{\psi}\left(\omega, r, \theta, k_{z m}\right) \mathrm{e}^{-\mathrm{i} k_{z m} z}, \\
& \chi(\omega, r, \theta, z)=\frac{2 \pi}{L} \sum_{m=-M}^{M} \hat{\chi}\left(\omega, r, \theta, k_{z m}\right) \mathrm{e}^{-\mathrm{i} k_{z m} z}, \\
& \phi_{f}(\omega, r, \theta, z)=\frac{2 \pi}{L} \sum_{m=-M}^{M} \hat{\phi}_{f}\left(\omega, r, \theta, k_{z m}\right) \mathrm{e}^{-\mathrm{i} k_{z m} z}
\end{aligned}
$$


with $k_{z m}$ being the axial wavenumber given by $k_{z m}=(2 \pi / L) m$. The distance $L$ must be sufficiently large to avoid spatial contamination from the virtual sources [10]. A similar procedure has been used by the authors to analyze wave propagation inside seismic prospecting boreholes [11] and the outdoor propagation of sound waves in the presence of obstacles [12].

\section{Analytical solution}

Consider a fluid-filled pipeline defined by the internal and external radii, $r_{A}$ and $r_{B}$, respectively, submerged in an inviscid compressible homogeneous fluid medium, as illustrated in Fig. 1. This pipeline is assumed to be illuminated by a harmonic dilatational source placed in the exterior fluid medium. The waves generated by this source propagate and hit the surface of the submerged pipe. After striking the external surface of the pipeline, part of the incident energy is reflected back into the exterior fluid medium, and the remaining energy is transmitted into the solid material, in the form of propagating body and guided waves. These waves continue to propagate and they eventually strike the inner surface of the pipe. Here, a similar phenomenon may occur, with part of the energy being transmitted to the inner fluid and the rest being reflected back to the elastic medium. This process will be repeated until all the energy is dissipated.

\subsection{The incident wave field}

The three-dimensional incident field for a point pressure source placed at $\left(x_{0}, 0,0\right)$ can be expressed by means of the velocity potential $\phi$, which must satisfy Eq. (3). A solution for this

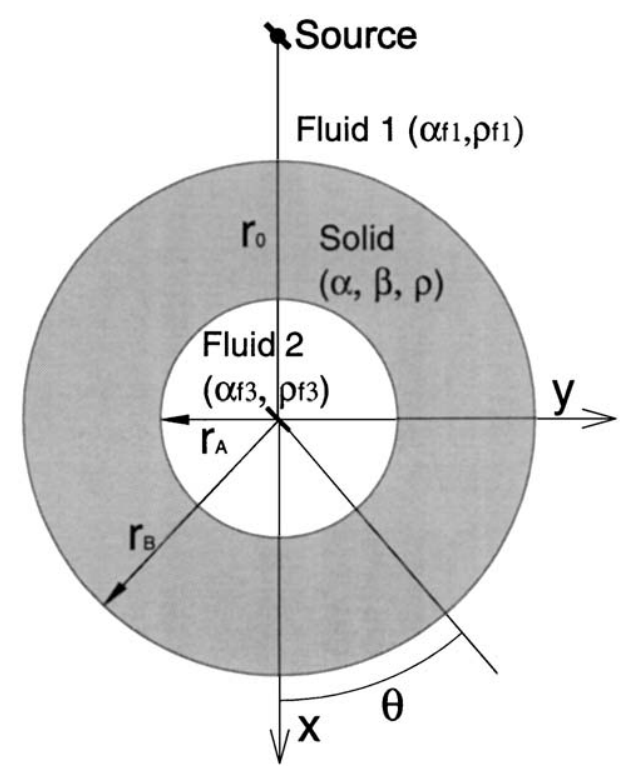

Fig. 1. Circular cylindrical pipeline submerged in a fluid medium. 
equation, as in [7], is

$$
\phi_{f i n c}(\omega, r)=\frac{A \mathrm{e}^{\mathrm{i}\left(\omega / \alpha_{f}\right)\left(\alpha_{f} t-r^{\prime}\right)}}{r^{\prime}}, \quad \text { with } r^{\prime}=\sqrt{\left(x-x_{0}\right)^{2}+y^{2}+z^{2}},
$$

where the subscript inc denotes the incident field, $A$ is the wave amplitude, $\alpha_{1}$ is the pressure wave velocity allowed in the exterior fluid medium, $r^{\prime}$ defines the distance between the source and the receiver and $\mathrm{i}=\sqrt{-1}$. The application of a Fourier transformation along the $z$ direction, allows the incident field to be expressed as a summation of 2-D sources, with different spatial wavenumbers

$$
\phi_{f i n c}(\omega, r, \theta, z)=\frac{2 \pi}{L} \sum_{m=-M}^{M} \hat{\phi}_{f i n c}\left(\omega, r, \theta, k_{z m}\right) \mathrm{e}^{-\mathrm{i} k_{z m} z}
$$

with

$$
\hat{\phi}_{f i n c}\left(\omega, r, \theta, k_{z m}\right)=\frac{-\mathrm{i} A}{2} \mathrm{H}_{0}^{(2)}\left(k_{\alpha_{f}} r^{\prime \prime}\right), \quad r^{\prime \prime}=\sqrt{\left(x-x_{0}\right)^{2}+y^{2}}, \quad k_{\alpha f}=\sqrt{\frac{\omega^{2}}{\alpha_{f}^{2}}-k_{z m}^{2}}\left(\operatorname{Im} k_{\alpha f}<0\right),
$$

where $\mathrm{H}_{n}^{(2)}(\ldots)$ represents the second Hankel functions of order $n$, and $L$ is the distance between virtual point sources equally spaced along $z$.

Using Graff's addition theorem [13], this field can be expressed in terms of waves centred on the axis of the pipeline, leading to the following expressions in cylindrical co-ordinates:

If the source is in Fluid 1 and $r<r_{0}$

$$
\phi_{i n c}=-\frac{\mathrm{i}}{2} \sum_{n=0}^{\infty}(-1)^{n} \varepsilon_{n} \mathrm{H}_{n}^{(2)}\left(k_{\alpha_{f 1}} r_{0}\right) \mathrm{J}_{n}\left(k_{\alpha_{f 1}} r\right) \cos (n \theta) \quad \text { with } k_{\alpha_{f 1}}=\sqrt{\frac{\omega^{2}}{\alpha_{f 1}^{2}}-k_{z m}^{2}} .
$$

If the source is in Fluid 2 and $r>r_{0}$

$$
\phi_{i n c}=-\frac{\mathrm{i}}{2} \sum_{n=0}^{\infty}(-1)^{n} \varepsilon_{n} \mathrm{~J}_{n}\left(k_{\alpha_{f 2}} r_{0}\right) \mathrm{H}_{n}^{(2)}\left(k_{\alpha_{f 2}} r\right) \cos (n \theta) \quad \text { with } k_{\alpha_{f 2}}=\sqrt{\frac{\omega^{2}}{\alpha_{f 2}^{2}}-k_{z m}^{2}}
$$

in which $r_{0}$ is the distance from the source to the axis of the pipeline, $\mathrm{J}_{n}(\ldots)$ are Bessel functions of order $n, \alpha_{f 1}$ and $\alpha_{f 2}$ are the pressure wave velocities for the outer and inner fluid, respectively, and

$$
\varepsilon_{n}= \begin{cases}\frac{1}{2} & \text { if } n=0 \\ 1 & \text { if } n \neq 0\end{cases}
$$

\subsection{The scattered wave field in the outer fluid}

The wavefield generated in the exterior fluid medium (Fluid 1) depends on waves coming from the external surface of the pipeline, which propagate away from it. The Hankel functions of order $n$ [7], $\mathrm{H}_{n}^{(1),(2)}(k r) \cos (n \theta)$, satisfy Eq. (5), representing cylindrical waves when combined with the implicit factor $\mathrm{e}^{-\mathrm{i} \omega t}$. It may be found that $\mathrm{H}_{n}^{(1)}(k r) \cos (n \theta)$ refers to converging or incoming waves, while $\mathrm{H}_{n}^{(2)}(k r) \cos (n \theta)$ corresponds to diverging or outgoing waves. So, when a cylindrical coordinate system is centred on the axis of the pipeline (see Fig. 1), the outgoing waves can be 
defined using the following velocity potential:

$$
\phi_{1}=\sum_{n=0}^{\infty} A_{n}^{(1)} \mathrm{H}_{n}^{(2)}\left(k_{\alpha_{f 1}} r\right) \cos (n \theta),
$$

where $A_{n}^{(1)}$ is an unknown potential amplitude.

\subsection{The scattered wave field in the solid material}

Two distinct groups of waves exist inside the elastic material of the pipeline, corresponding to waves generated at the external surface and travelling inwards, and to waves generated at the internal surface of the pipe that travel outwards. Using the co-ordinate system described above, each of these groups can be described by one dilatational and two shear potentials, as in Eqs. (4). For the waves generated at the external boundary, the corresponding potentials are equivalent to standing wavefields inside a circular cylinder, resulting from the sum of the incoming and outgoing waves [7], leading to the definition of the following potentials, which satisfy Eqs. (4):

$$
\begin{aligned}
& \phi_{2}=\sum_{n=0}^{\infty} A_{n}^{(2)} \mathrm{J}_{n}\left(k_{\alpha} r\right) \cos (n \theta), \\
& \psi_{2}=\sum_{n=0}^{\infty} A_{n}^{(3)} \mathrm{J}_{n}\left(k_{\beta} r\right) \sin (n \theta), \\
& \chi_{2}=\sum_{n=0}^{\infty} A_{n}^{(4)} \mathrm{J}_{n}\left(k_{\beta} r\right) \cos (n \theta),
\end{aligned}
$$

where

$$
k_{\alpha}=\sqrt{\frac{\omega^{2}}{\alpha^{2}}-k_{z m}^{2}}, \quad k_{\beta}=\sqrt{\frac{\omega^{2}}{\beta^{2}}-k_{z m}^{2}}
$$

and $A_{n}^{(2)}, A_{n}^{(3)}$ and $A_{n}^{(4)}$ are unknown potential amplitudes.

For the waves generated at the internal boundary, the corresponding potentials are equivalent to diverging wavefields, which can be defined by the potentials

$$
\begin{aligned}
& \phi_{3}=\sum_{n=0}^{\infty} A_{n}^{(5)} \mathrm{H}_{n}^{(2)}\left(k_{\alpha} r\right) \cos (n \theta), \\
& \psi_{3}=\sum_{n=0}^{\infty} A_{n}^{(6)} \mathrm{H}_{n}^{(2)}\left(k_{\beta} r\right) \sin (n \theta), \\
& \chi_{3}=\sum_{n=0}^{\infty} A_{n}^{(7)} \mathrm{H}_{n}^{(2)}\left(k_{\beta} r\right) \cos (n \theta),
\end{aligned}
$$

where $A_{n}^{(5)}, A_{n}^{(6)}$ and $A_{n}^{(7)}$ are unknown potential amplitudes. 


\subsection{The scattered wave field in the inner fluid}

In the inner fluid (Fluid 2), the wavefield depends only on waves coming from the internal surface of the pipeline, and thus only inward propagating waves are generated. The corresponding velocity potential, which satisfies Eq. (5), is:

$$
\phi_{4}=\sum_{n=0}^{\infty} A_{n}^{(8)} \mathbf{J}_{n}\left(k_{\alpha_{f 2}} r\right) \cos (n \theta),
$$

where $A_{n}^{(8)}$ is an unknown potential amplitude.

The unknown coefficients $A_{n}^{(j)}(j=1,8)$ are determined by imposing the required boundary conditions. For the present case, the boundary conditions are the continuity of normal displacements and stresses and null tangential stresses on the two solid-fluid interfaces. A more detailed explanation of the final equation system can be found in the appendix.

\section{Time responses}

The results (displacements and stresses) in the spatial-temporal domain can be calculated from the frequency domain responses, by applying a numerical fast inverse Fourier transform in $\omega$. In this process, a source with a temporal variation given by a Ricker pulse is modelled. The rapid decay of this pulse in both the time and frequency domains reduces the computational effort, and also makes it easier to interpret the time responses.

In the frequency domain, the Ricker wavelet function is defined by

$$
U(\omega)=A\left[2 \sqrt{\pi} t_{o} \mathrm{e}^{-\mathrm{i} \omega t_{s}}\right] \Omega^{2} \mathrm{e}^{-\Omega^{2}},
$$

where $A$ is the amplitude, $\Omega=\omega t_{o} / 2, t_{s}$ is the time when the maximum occurs, while $\pi t_{o}$ is the characteristic period of the wavelet.

This procedure allows the definition of a total time window $T=2 \pi / \Delta \omega(\Delta \omega$ being the frequency step) for the time domain analysis. Pulses arriving at the receivers at times later than $T$ will appear again in the beginning of this window, generating the so-called aliasing phenomenon. The contribution of these pulses can be greatly attenuated if the frequency axis is shifted slightly downward, that is, by using complex frequencies with a small imaginary part of the form $\omega_{c}=$ $\omega-$ i $\eta$ (with $\eta=0.7 \Delta \omega$ ) (e.g., Ref. [14]). For the analysis in the time domain, this shift must be taken into account by rescaling the response using an exponential window $\mathrm{e}^{\eta t}[15]$.

\section{Numerical examples}

A set of numerical examples is now presented, modelling an infinite circular pipeline with an internal radius of $1.00 \mathrm{~m}$ and a wall thickness of either 0.03 or $0.10 \mathrm{~m}$, submerged in a homogeneous fluid medium. The fluid filling the pipeline is assumed to have the same properties as the host medium, allowing a pressure wave propagation velocity of $1500 \mathrm{~m} / \mathrm{s}$, and exhibiting a density of $\rho=1000 \mathrm{~kg} / \mathrm{m}^{3}$. The elastic material of the pipeline is steel, with a Poisson ratio of $v=0.3$, a density of $\rho=7850 \mathrm{~kg} / \mathrm{m}^{3}$ and Young's modulus $E=210.6 \mathrm{GPa}$. These mechanical 


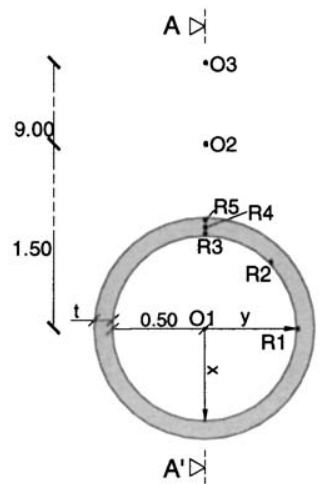

(a)

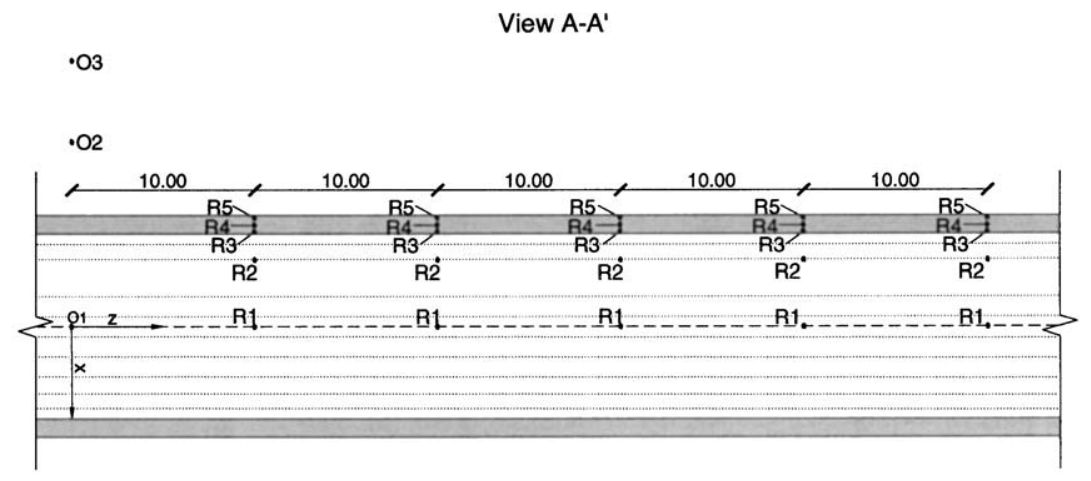

(b)

Fig. 2. Geometry of the model: (a) front view; (b) side view.

properties allow propagation velocities for the $\mathrm{P}$ and $\mathrm{S}$ waves of 6009 and $3212 \mathrm{~m} / \mathrm{s}$ respectively. Point pressure loads are placed in either the exterior or the interior fluid to excite the dynamic system. Stresses in the elastic material are calculated at lines of five receivers placed along the $z$ direction, and positioned in the dynamic system as shown in Fig. 2.

All the computations were performed for frequencies ranging from 4.0 to $1024.0 \mathrm{~Hz}$, with increments of $4.0 \mathrm{~Hz}$. This frequency step of $4.0 \mathrm{~Hz}$ determines a maximum analysis time of $0.25 \mathrm{~s}$ for the time domain responses. The time domain responses presented are computed by means of an inverse Fourier transformation, assuming the source generates a Ricker pulse with a central frequency of $350.0 \mathrm{~Hz}$.

As explained before, the response for each frequency is computed as a summation of simpler 2-D problems, each solved for an individual value of the axial wavenumber $k_{z}$. The final solution can be seen as the result of the contribution of different modes associated with the propagation of different wave types. The different wave types in the frequency vs. axial-wavenumber domain are more easily recognized and identified, as they occupy specific sub-domains, according to their wave velocity. Both time and frequency vs. $k_{z}$ domain results are used to illustrate the main wave features. The normal stresses $\sigma_{r r}$ are used to illustrate the main features of the responses. Similar conclusions would be obtained by analyzing other stress distributions.

The first model corresponds to a circular cylindrical pipeline with a wall $0.03 \mathrm{~m}$ thick. Fig. 3 gives both the frequency vs. $k_{z}$ response and the time domain responses at receivers $\mathrm{R} 1$, placed close to the inner surface of the pipeline, when a point pressure load is excited at position O1, in the centre of the pipeline. Results in the frequency vs. $k_{z}$ domain (Fig. 3a) are presented using a grey scale, ranging from white to black as the amplitude of the stresses decreases. The frequency domain results obtained at these receivers show the existence of different wave modes propagating in the dynamic system. These modes correspond to the guided and body waves generated by the excitation source. Some of these modes exhibit an azimuthal variation. In this work they are referred to as "Ai", where $\mathrm{i}$ indicates the variation of the mode with azimuth.

Pulses associated with waves travelling in the fluid at a velocity of $1500 \mathrm{~m} / \mathrm{s}$ are clearly visible (identified by "F" in Fig. 3b). Additionally, two modes are excited (labelled in Fig. 3 as "A0" and "TP"). The first mode is axisymmetric; it is dispersive and exhibits lower group and phase 

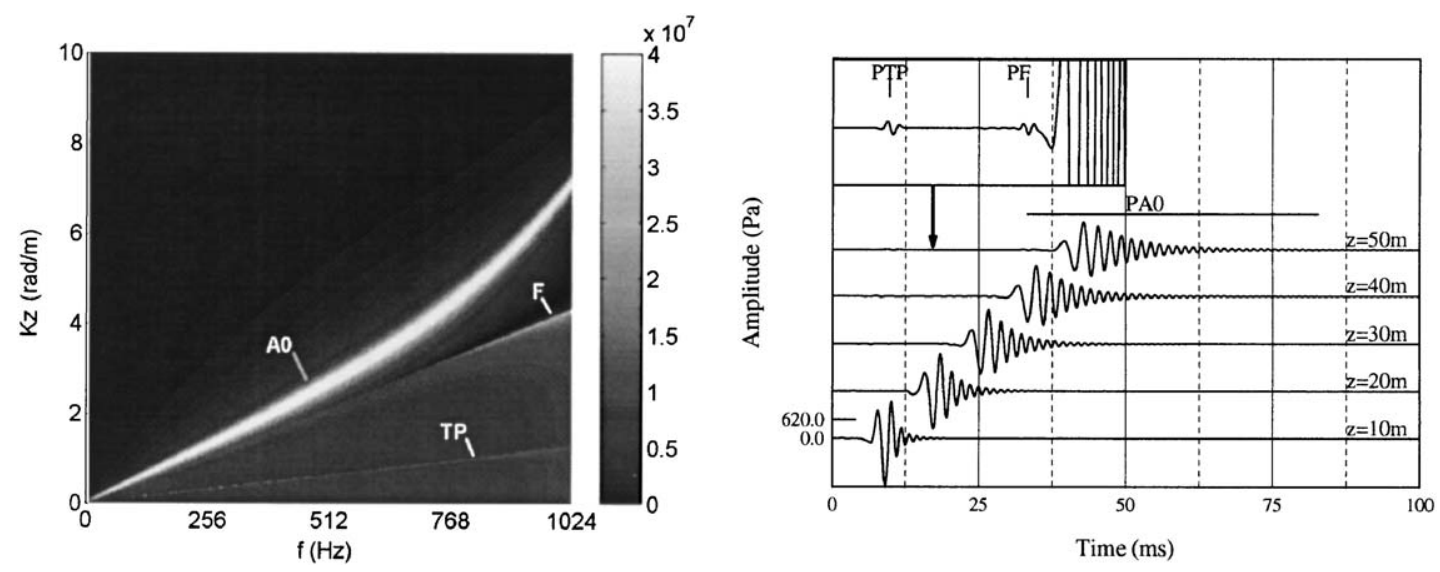

Fig. 3. Frequency and time domain response at receivers R1 due to a pressure load located at O1.

velocities than the fluid pressure wave velocity ("A0"). It seems to exist in the full frequency range. The second mode appears to be only slightly dispersive. It exhibits higher propagation velocities and is associated with waves that exist in thin plates and shells. According to Graff [16], this "thin plate velocity" is given by $\sqrt{E /\left(1-v^{2}\right) \rho}$. The mechanical properties ascribed to the elastic medium allow the "thin plate velocity" to be approximately $5429 \mathrm{~m} / \mathrm{s}$. The calculation using this expression does not take into account the solid/fluid coupling, and thus there is a discrepancy between this result and the group velocities observed in the results presented here $(\approx 5200 \mathrm{~m} / \mathrm{s})$.

The main features described can also be identified in the time domain responses of Fig. 3 . The first pulse arriving at the receivers corresponds to waves travelling at higher velocities (labelled "PTP"). These waves are associated with the "TP" mode, and travel at a velocity of approximately $5200 \mathrm{~m} / \mathrm{s}$. A second pulse can be detected in the time responses, corresponding to waves travelling at the fluid velocity, and labelled "PF". There follows a set of pulses associated with the "A0" normal mode (labelled "PA0"). Since "A0" is a highly dispersive mode, its contribution appears in the time response as a sequence of pulses that start to arrive after the waves that travel at the pressure wave velocity of the fluid.

Fig. 4 gives both the frequency spectra and the time domain responses for the normal stresses $\sigma_{r r}$ at receivers $\mathrm{R} 1, \mathrm{R} 2$ and $\mathrm{R} 3$, placed close to the inner surface of the pipeline, when a point pressure load is excited at position $\mathrm{O} 2,1.5 \mathrm{~m}$ from the centre of the pipeline.

The response registered at receivers R1 (Fig. 4a), placed over the neutral axis of the system (the horizontal line passing through the centre of the pipeline), indicates the existence of several types of waves, which correspond to the body waves and waves associated with different normal modes of the dynamic system.

Again, pulses associated with waves travelling in the fluid at a velocity of $1500 \mathrm{~m} / \mathrm{s}$, are clearly visible (identified by " $F$ " in the figure). Receivers placed at this position easily detect the two modes described above (labelled in Fig. 4a as "TP" and "A0"). Non-axisymmetric modes are also excited here, corresponding to screw waves (identified as "A2"), while there are no bending waves ("A1") since the receivers are over the neutral axis. These non-axisymmetric modes are also dispersive. Other non-axisymmetric modes exist that correspond to modes of a higher azimuthal 

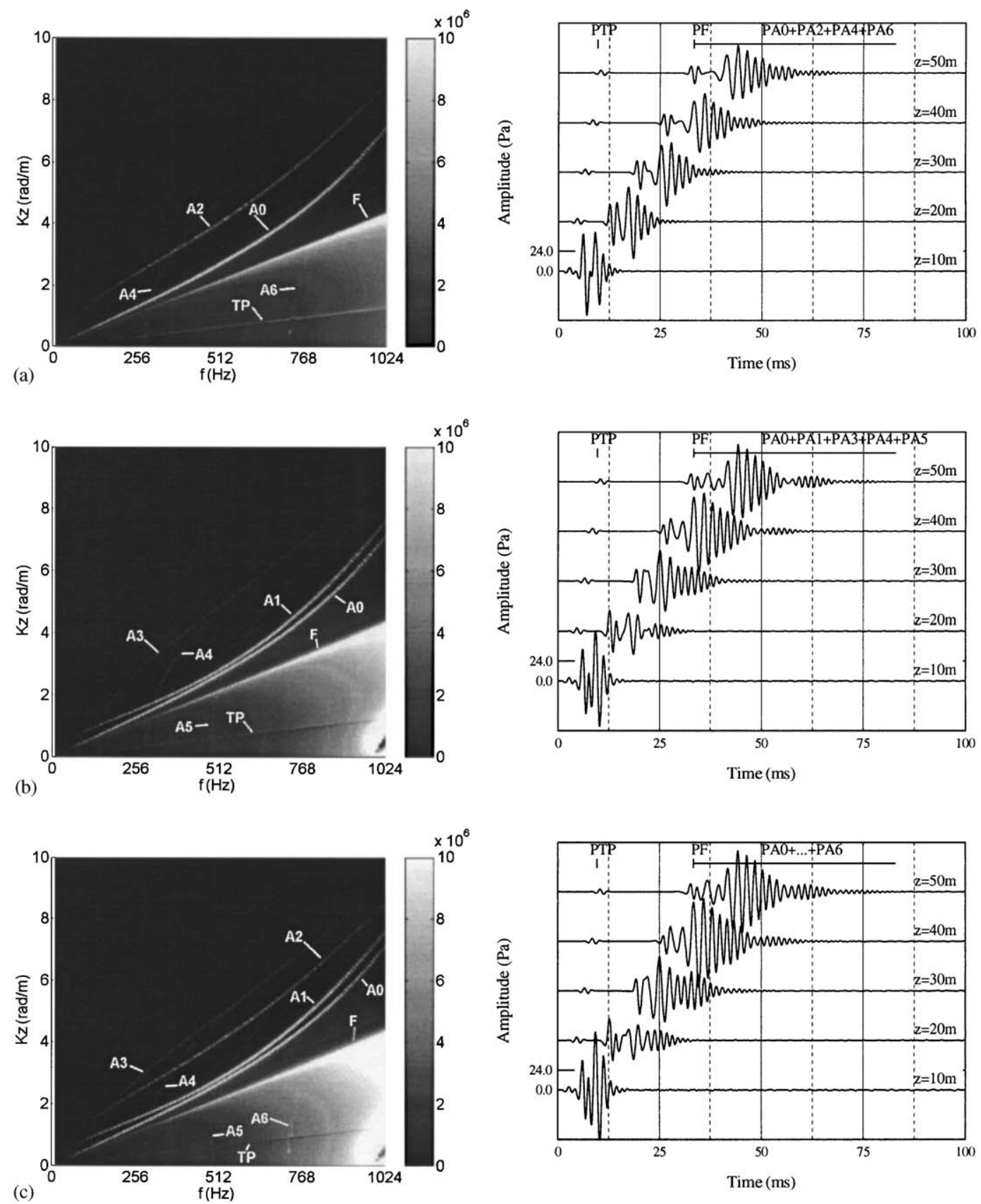

Fig. 4. Frequency and time domain responses due to a pressure load located at O2: (a) receivers R1; (b) receivers R2; (c) receivers $\mathrm{R} 3$.

order (labelled "Ai"). However, these are only visible at receivers placed away from the nodal line of the mode. The R1 receivers only record the "A4" and "A6" modes for the frequency range analyzed, and these exhibit weak amplitudes and well-defined cut-off frequencies. 
The features described here for the frequency response can be observed in the time domain responses, where the arrival times of the different waves can be identified. Again, it is possible to identify the arrival times of the pulses associated with the "TP" (labelled "PTP") and "F" (labelled "PF") waves. Following these, a sequence of pulses ("PA0") caused by the excitation of the dispersive "A0" normal mode arrives at the receivers. The contribution of the screw waves ("PA2") and of the "A4" and "A6" modes is not clear in the time responses, since these normal modes exhibit very low amplitudes compared with the "A0" and "TP" waves.

The results computed at receivers R2 are presented in Fig. 4b. The modes referred to earlier ("F", "A0" and "TP") are also detected at these receivers, as was expected. However, the screw mode ("A2") previously observed does not exist at this position, since it makes null contribution for receivers placed over a line that passes through the centre of the pipeline and makes an angle of $45^{\circ}$ with the horizontal axis. Since the receivers are not over the neutral axis, the bending waves ("A1") contribute to the response, appearing as a dispersive mode. At low frequencies these waves exhibit group velocities closer to the pressure wave velocity in the fluid, while at high frequencies the group velocity becomes slower. Modes of higher azimuthal order, namely the "A3", "A4" and "A5" modes, also contribute to the response, but with lower amplitudes. The time response also reveals the existence of the bending mode ("PA1"). Due to its dispersive nature, it appears in the responses as a pack of waves that start arriving after the waves associated with the pressure wave velocity of the fluid, together with waves associated with the "A0" mode. Since these "A1" waves reach group velocities below those of the "A0" mode, in the frequency range analyzed, they are still visible after the last arrivals associated with the first axisymmetric mode. Due to their very low amplitude, the contribution of higher order modes is not visible in the time response.

Fig. $4 \mathrm{c}$ shows the results computed at receivers R3. These receivers record the contribution of both the axisymmetric and nonaxisymmetric modes. The axisymmetric ("A0"), bending ("A1") and screw modes ("A2") can now be identified in the frequency response, together with all the higher order modes identified before. The contribution of waves travelling with the fluid velocity ("F") and with the "thin plate velocity" ("TP") is also clearly visible. Again, in the time response, the pulses arriving at the receivers associated with the "TP" ("PTP") and "F" ("PF") modes can be identified. However, the superposition of modes with slower velocities makes it difficult to fully identify the contribution of each of the remaining modes to the time response.

The response of the same model has also been analyzed for receivers placed over the midsurface and close to the external boundary of the elastic material, assuming the source to be placed at position O2. Fig. 5a illustrates the stresses $\sigma_{r r}$ calculated at receivers R4, placed on the mid-surface of the pipeline and over the line connecting the source and the centre of the pipeline. The responses registered at these receivers are more complex than the ones described before, since the contribution of normal modes of higher azimuthal order is enhanced. Since their amplitude is much higher than before, it is now easy to see that they exhibit well-defined cut-off frequencies. The modes identified in this figure are the same as those found before at receivers R3, corresponding to dispersive axisymmetric ("A0"), bending ("A1"), screw ("A2"), and normal modes of higher order ("A3", "A4", "A5" and "A6") that are generated in the dynamic system. Very slow group velocities characterize all these later modes at low frequencies. Consequently, a set of pulses appears at later times in the time domain responses. The high number of modes excited with slower velocities means that it is not possible to distinguish their individual contributions to the final response. When the receivers are placed close to the external surface 

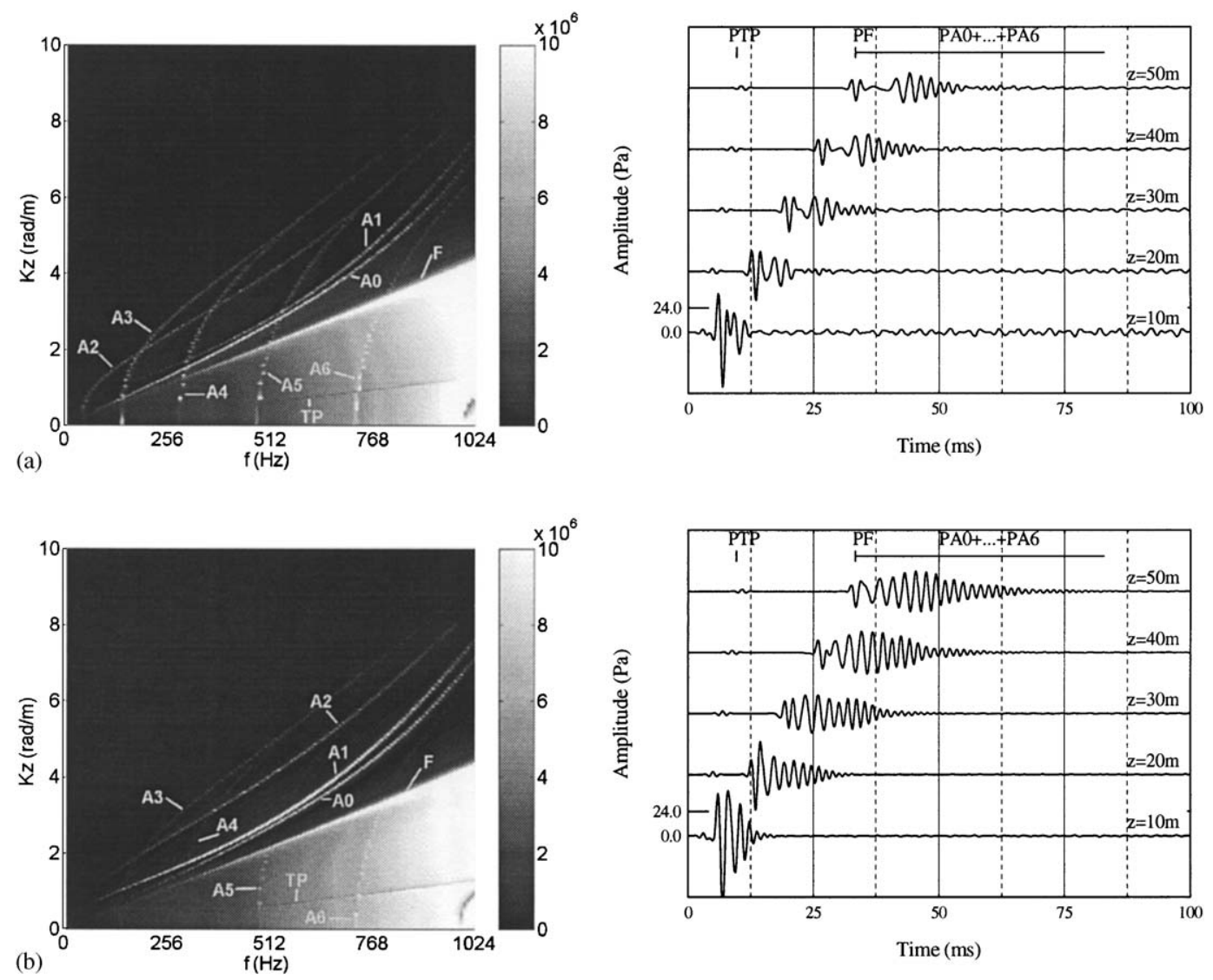

Fig. 5. Frequency and time domain responses due to a pressure load located at O2: (a) receivers R4; (a) receivers R5.

(Fig. 5b), the normal modes of higher order become less important, and thus the response approaches the one obtained for receivers R3. Analyzing the responses obtained at receivers 4 and 5 , it can be said that the contribution of the bending ("A1") and screw ("A2") modes to the response appears to be less significant when the receiver is located further away from the surface of the elastic material.

A second simulation was performed for a point source placed at position $\mathrm{O} 3,10.5 \mathrm{~m}$ away from the axis of the pipeline. Fig. 6 presents the stresses computed at receivers R3, over the system's axis of symmetry and close to the internal wall of the pipeline. The response recorded at these receivers is much simpler than the ones described previously. The different normal modes are still excited, but now they exhibit much lower amplitudes. Thus, the response is dominated by the waves travelling along the fluid and by those moving inside the pipeline wall with the so-called "thin plate velocity". In the time responses, this behaviour is even more evident, and only pulses that correspond to those two types of waves are clearly visible. It can be seen that the amplitude of the response registered at receivers placed further away from the plane containing the source decreases faster than in the previous cases. The spreading of energy in the fluid media and the low contribution of the guided modes explain this behaviour. 

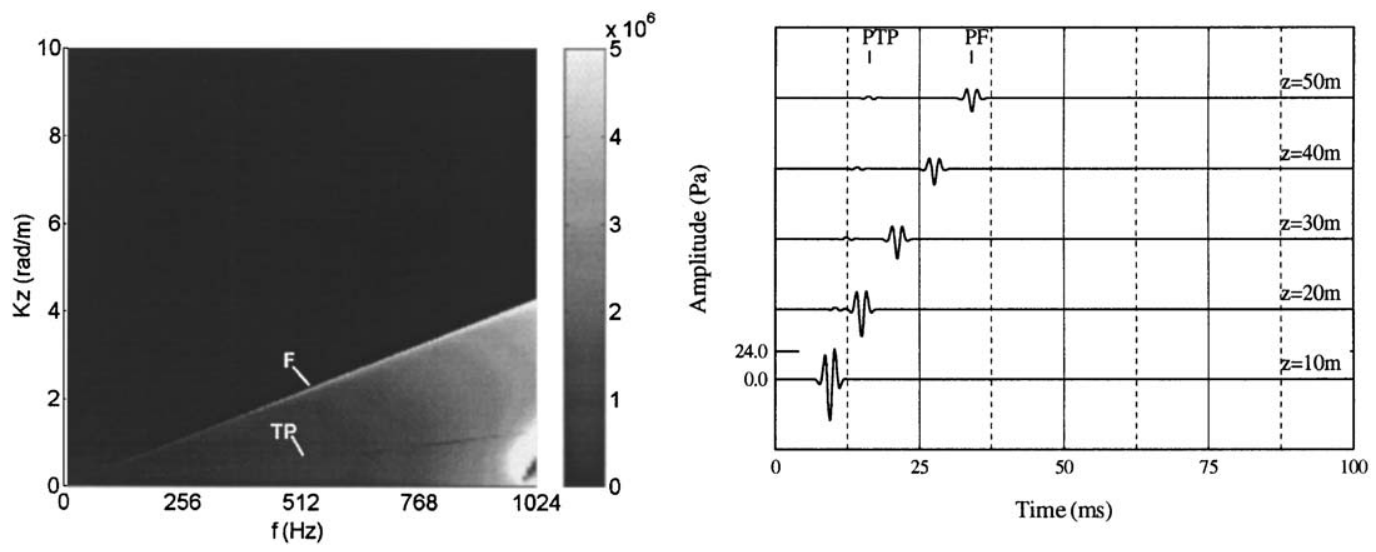

Fig. 6. Frequency and time domain response at receivers R3 due to a source placed at O3.

One last simulation was performed for a pipeline with a wall $0.10 \mathrm{~m}$ thick Fig. 7 presents the results obtained at receivers $\mathrm{R} 1, \mathrm{R} 2$ and $\mathrm{R} 3$, for an excitation source placed at $\mathrm{O} 2$. The frequency domain responses at all the receivers clearly indicate the contribution of different wave types, as observed before for $t=0.03 \mathrm{~m}$. All receivers register the contribution of waves travelling at the pressure wave velocity allowed by the fluid medium and at the so-called "thin-plate velocity". An axisymmetric mode ("A0") is also visible at the full set of receivers, existing for the full frequency range. Furthermore, different non-axisymmetric modes are detected at each receiver. As before, responses recorded at receivers R1 reveal the presence of screw waves (“A2"), while bending ("A1") and third order ("A3") normal modes have a null contribution at these receivers. By contrast, at receivers $\mathrm{R} 2$ bending waves are visible ("A1") together with the normal mode of third order ("A3"). Receivers R3 register the contribution of the three non-axisymmetric normal modes described. Comparing these results with the corresponding ones in Fig. 3 we find that there is a substantial change in the group and phase velocities of the different normal modes. With the thicker wall, the bending and the screw wave group velocities seem to approach the group velocity of the axisymmetric normal mode as the frequency increases. For low frequencies, the group velocity of the bending mode even exceeds it. In general, it is possible to observe an increase in the cut-off frequency of the higher normal modes, together with an increase in their phase velocities, particularly for higher frequencies. This increase in velocities results in the time responses showing that the slower pulses arrive earlier than before. One can also see that the bending waves now start arriving before the pulse that travels at the pressure wave velocity of the fluid.

\section{Conclusions}

The stresses generated in an infinite straight pipeline submerged in a homogeneous fluid medium and subjected to the incidence of pressure waves generated by point source have been analyzed. The section of the pipeline was assumed to be defined by two concentric circumferences, and was modelled as an elastic material. The computations were performed in the frequency 

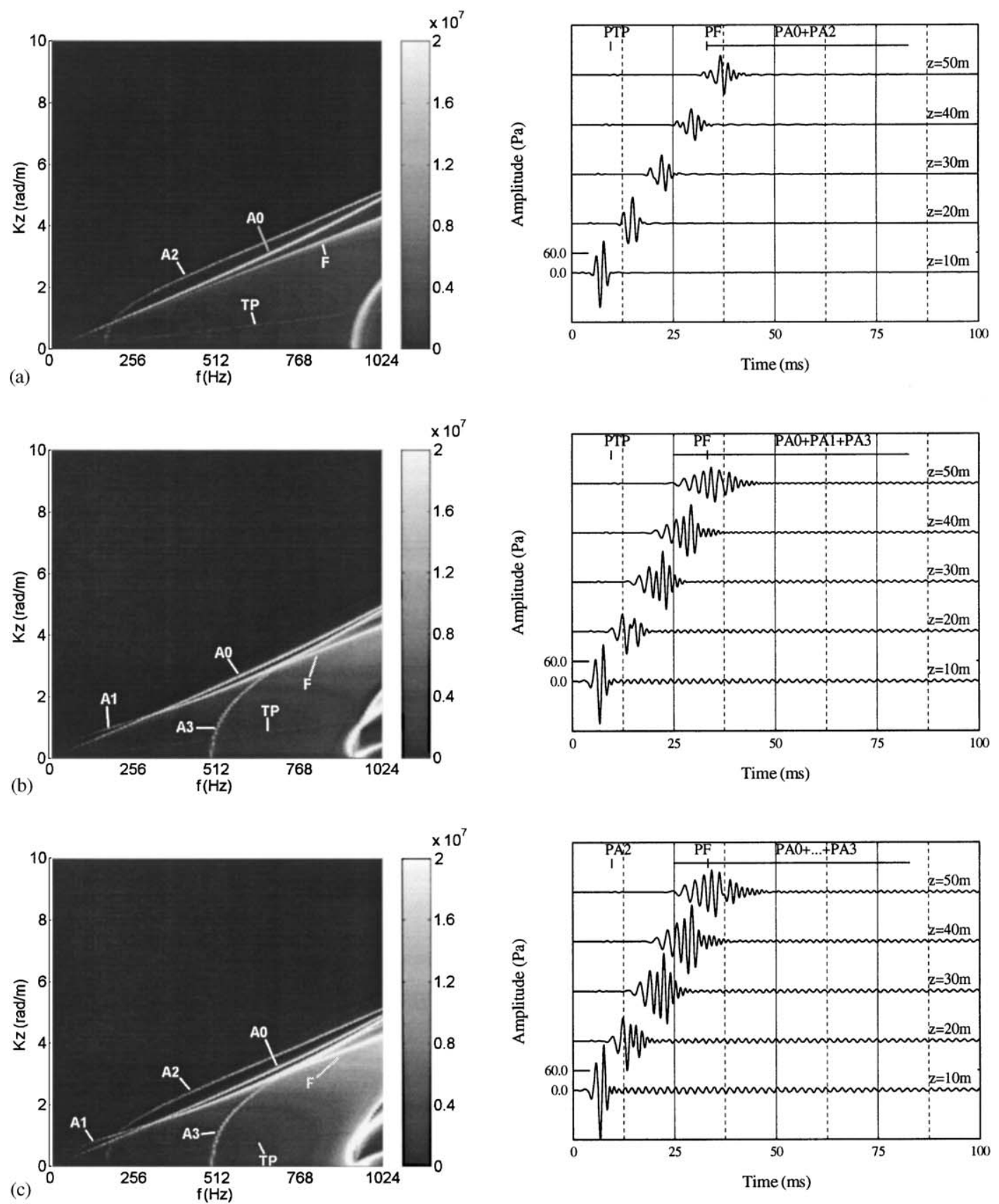

Fig. 7. Frequency and time domain response when the pipeline has a thickness of $0.10 \mathrm{~m}$ and a pressure load is located at $\mathrm{O} 2$ : (a) receivers $\mathrm{R} 1$; (b) receivers $\mathrm{R} 2$; (c) receivers $\mathrm{R} 3$.

domain using analytical solutions that take the full solid-fluid interaction into account. Time domain responses were also calculated, allowing the main features of the wave propagation to be identified. 
The influence of the position of the receiver on the final response registered was analyzed, and it was found that the contribution of the different normal modes does depend on receiver position. It can be said that the stresses registered at each point inside the elastic material of the pipeline are strongly dependent on both the body waves generated and the stresses provided by the individual contribution of the different guided wave modes at each position.

The different simulations showed that the amplitude of the excited normal modes is governed to a great extent by the distance between the source and the pipeline. The contribution of the guided waves decreases markedly when the source is placed further away from the pipeline. For this case, however, the contributions of the waves associated both with waves travelling at the pressure wave velocity of the fluid and with those moving in the pipeline wall at the so-called "thin plate velocity" were still significant. The thickness of the pipeline cross-section was also found to influence the properties of the modes excited in the system, particularly their velocity and cut-off frequency. The dispersion wave velocities of the different modes were found to be influenced by the presence of the fluids.

\section{Appendix}

The eight potentials defined give rise to a system of eight equations with eight unknowns, which yields the coefficients $A_{n}^{(i)}(i=1, \ldots, 8)$. This system is built so as to allow the establishment of boundary conditions of null tangential stresses and the continuity of normal displacements and stresses in the solid-fluid interfaces:

$$
\left[\begin{array}{ccc}
a_{11} & . . & a_{18} \\
: & & : \\
a_{81} & . . & a_{88}
\end{array}\right]\left[\begin{array}{c}
A_{n}^{(1)} \\
: \\
A_{n}^{(8)}
\end{array}\right]=\left[\begin{array}{c}
b_{1} \\
: \\
b_{8}
\end{array}\right] .
$$

Terms $a_{i j}$ are defined as

Outer boundary:

$$
\begin{gathered}
a_{11}=\lambda_{f 1}\left(k_{\alpha f 1}^{2}+k_{z}^{2}\right) \mathrm{H}_{n}^{(2)}\left(k_{\alpha_{f 1}} r_{B}\right), \\
a_{12}=\frac{2 \mu}{r_{B}^{2}}\left[\left(n^{2}-n-\frac{\omega^{2} r_{B}^{2}}{2 \beta^{2}}+k_{z}^{2} r_{B}^{2}\right) \mathrm{J}_{n}\left(k_{\alpha} r_{B}\right)+\left(k_{\alpha} r_{B}\right) \mathrm{J}_{n+1}\left(k_{\alpha} r_{B}\right)\right], \\
a_{13}=\frac{2 \mu}{r_{B}^{2}}\left[\left(-n+n^{2}\right) \mathrm{J}_{n}\left(k_{\beta} r_{B}\right)-\left(n k_{\beta} r_{B}\right) \mathrm{J}_{n+1}\left(k_{\beta} r_{B}\right)\right], \\
a_{14}=-\mathrm{i} k_{z} \frac{2 \mu}{r_{B}^{2}}\left[\left(-n+n^{2}-k_{\beta}^{2} r_{B}^{2}\right) \mathrm{J}_{n}\left(k_{\beta} r_{B}\right)+\left(k_{\beta} r_{B}\right) \mathrm{J}_{n+1}\left(k_{\beta} r_{B}\right)\right], \\
a_{15}=\frac{2 \mu}{r_{B}^{2}}\left[\left(n^{2}-n-\frac{\omega^{2} r_{B}^{2}}{2 \beta^{2}}+k_{z}^{2} r_{B}^{2}\right) \mathrm{H}_{n}^{(2)}\left(k_{\alpha} r_{B}\right)+\left(k_{\alpha} r_{B}\right) \mathrm{H}_{n+1}^{(2)}\left(k_{\alpha} r_{B}\right)\right], \\
a_{16}=\frac{2 \mu}{r_{B}^{2}}\left[\left(-n+n^{2}\right) \mathrm{H}_{n}^{(2)}\left(k_{\beta} r_{B}\right)-\left(n k_{\beta} r_{B}\right) \mathrm{H}_{n+1}^{(2)}\left(k_{\beta} r_{B}\right)\right],
\end{gathered}
$$




$$
\begin{aligned}
& a_{17}=-\mathrm{i} k_{z} \frac{2 \mu}{r_{B}^{2}}\left[\left(-n+n^{2}-k_{\beta}^{2} r_{B}^{2}\right) \mathrm{H}_{n}^{(2)}\left(k_{\beta} r_{B}\right)+\left(k_{\beta} r_{B}\right) \mathrm{H}_{n+1}^{(2)}\left(k_{\beta} r_{B}\right)\right], \\
& a_{18}=0, \\
& a_{21}=0, \\
& a_{22}=\left[\left(n-n^{2}\right) \mathrm{J}_{n}\left(k_{\alpha} r_{B}\right)+\left(n k_{\alpha} r_{B}\right) \mathrm{J}_{n+1}\left(k_{\alpha} r_{B}\right)\right], \\
& a_{23}=\left[\left(n-n^{2}+\frac{k_{\beta}^{2} r_{B}^{2}}{2}\right) \mathbf{J}_{n}\left(k_{\beta} r_{B}\right)+\left(-k_{\beta} r_{B}\right) \mathbf{J}_{n+1}\left(k_{\beta} r_{B}\right)\right], \\
& a_{24}=-n \mathrm{i} k_{z}\left[(-n+1) \mathrm{J}_{n}\left(k_{\beta} r_{B}\right)+\left(k_{\beta} r_{B}\right) \mathrm{J}_{n+1}\left(k_{\beta} r_{B}\right)\right] \text {, } \\
& a_{25}=\left[\left(n-n^{2}\right) \mathrm{H}_{n}^{(2)}\left(k_{\alpha} r_{B}\right)+\left(n k_{\alpha} r_{B}\right) \mathrm{H}_{n+1}^{(2)}\left(k_{\alpha} r_{B}\right)\right], \\
& a_{26}=\left[\left(n-n^{2}+\frac{k_{\beta}^{2} r_{B}^{2}}{2}\right) \mathrm{H}_{n}^{(2)}\left(k_{\beta} r_{B}\right)+\left(-k_{\beta} r_{B}\right) \mathrm{H}_{n+1}^{(2)}\left(k_{\beta} r_{B}\right)\right], \\
& a_{27}=-n \mathrm{i} k_{z}\left[(-n+1) \mathrm{H}_{n}^{(2)}\left(k_{\beta} r_{B}\right)+\left(k_{\beta} r_{B}\right) \mathrm{H}_{n+1}^{(2)}\left(k_{\beta} r_{B}\right)\right] \text {, } \\
& a_{28}=0, \\
& a_{31}=0, \\
& a_{32}=-\mathrm{i} k_{z}\left[\left(n r_{B}\right) \mathrm{J}_{n}\left(k_{\alpha} r_{B}\right)-\left(k_{\alpha} r_{B}^{2}\right) \mathbf{J}_{n+1}\left(k_{\alpha} r_{B}\right)\right] \text {, } \\
& a_{33}=\left[\frac{-\mathrm{i} k_{z}}{2} n r_{B} \mathrm{~J}_{n}\left(k_{\beta} r_{B}\right)\right] \text {, } \\
& a_{34}=\left\{\frac{1}{2 r_{B}}\left(k_{\beta}^{2} r_{B}^{2}-k_{z}^{2} r_{B}^{2}\right)\left[n \mathbf{J}_{n}\left(k_{\beta} r_{B}\right)-\left(k_{\beta} r_{B}\right) \mathbf{J}_{n+1}\left(k_{\beta} r_{B}\right)\right]\right\}, \\
& a_{35}=-\mathrm{i} k_{z}\left[\left(n r_{B}\right) \mathrm{H}_{n}^{(2)}\left(k_{\alpha} r_{B}\right)-\left(k_{\alpha} r_{B}^{2}\right) \mathrm{H}_{n+1}^{(2)}\left(k_{\alpha} r_{B}\right)\right], \\
& a_{36}=\left[\frac{-\mathrm{i} k_{z}}{2} n r_{B} \mathrm{H}_{n}^{(2)}\left(k_{\beta} r_{B}\right)\right] \text {, } \\
& a_{37}=\left\{\frac{1}{2 r_{B}}\left(k_{\beta}^{2} r_{B}^{2}-k_{z}^{2} r_{B}^{2}\right)\left[n \mathrm{H}_{n}^{(2)}\left(k_{\beta} r_{B}\right)-\left(k_{\beta} r_{B}\right) \mathrm{H}_{n+1}^{(2)}\left(k_{\beta} r_{B}\right)\right]\right\} \text {, } \\
& a_{38}=0 \text {, } \\
& a_{41}=-\left[\frac{n}{r_{B}} \mathrm{H}_{n}^{(2)}\left(k_{\alpha_{f 1}} r_{B}\right)-k_{\alpha_{f 1}} \mathrm{H}_{n+1}^{(2)}\left(k_{\alpha_{f 1}} r_{B}\right)\right] \text {, }
\end{aligned}
$$




$$
\begin{gathered}
a_{42}=\left[\frac{n}{r_{B}} \mathrm{~J}_{n}\left(k_{\alpha} r_{B}\right)-k_{\alpha} \mathrm{J}_{n+1}\left(k_{\alpha} r_{B}\right)\right], \\
a_{43}=\left[\frac{n}{r_{B}} \mathrm{~J}_{n}\left(k_{\beta} r_{B}\right)\right], \\
a_{44}=-\mathrm{i} k_{z}\left[\frac{n}{r_{B}} \mathrm{~J}_{n}\left(k_{\beta} r_{B}\right)-k_{\beta} \mathrm{J}_{n+1}\left(k_{\beta} r_{B}\right)\right], \\
a_{45}=\left[\frac{n}{r_{B}} \mathrm{H}_{n}^{(2)}\left(k_{\alpha} r_{B}\right)-k_{\alpha} \mathrm{H}_{n+1}^{(2)}\left(k_{\alpha} r_{B}\right)\right], \\
a_{46}=\left[\frac{n}{r_{B}} \mathrm{H}_{n}^{(2)}\left(k_{\beta} r_{B}\right)\right], \\
a_{47}=-\mathrm{i} k_{z}\left[\frac{n}{r_{B}} \mathrm{H}_{n}^{(2)}\left(k_{\beta} r_{B}\right)-k_{\beta} \mathrm{H}_{n+1}^{(2)}\left(k_{\beta} r_{B}\right)\right], \\
a_{48}=0 .
\end{gathered}
$$

Inner boundary:

$$
\begin{gathered}
a_{51}=0, \\
a_{52}=\frac{2 \mu}{r_{A}^{2}}\left[\left(n^{2}-n-\frac{\omega^{2} r_{A}^{2}}{2 \beta^{2}}+k_{z}^{2} r_{A}^{2}\right) \mathrm{J}_{n}\left(k_{\alpha} r_{A}\right)+\left(k_{\alpha} r_{A}\right) \mathrm{J}_{n+1}\left(k_{\alpha} r_{A}\right)\right] \\
a_{53}=\frac{2 \mu}{r_{A}^{2}}\left[\left(-n+n^{2}\right) \mathrm{J}_{n}\left(k_{\beta} r_{A}\right)-\left(n k_{\beta} r_{A}\right) \mathrm{J}_{n+1}\left(k_{\beta} r_{A}\right)\right], \\
a_{54}=-\mathrm{i} k_{z} \frac{2 \mu}{r_{A}^{2}}\left[\left(-n+n^{2}-k_{\beta}^{2} r_{A}^{2}\right) \mathrm{J}_{n}\left(k_{\beta} r_{A}\right)+\left(k_{\beta} r_{A}\right) \mathrm{J}_{n+1}\left(k_{\beta} r_{A}\right)\right], \\
a_{55}=\frac{2 \mu}{r_{A}^{2}}\left[\left(n^{2}-n-\frac{\omega^{2} r_{A}^{2}}{2 \beta^{2}}+k_{z}^{2} r_{A}^{2}\right) \mathrm{H}_{n}^{(2)}\left(k_{\alpha} r_{A}\right)+\left(k_{\alpha} r_{A}\right) \mathrm{H}_{n+1}^{(2)}\left(k_{\alpha} r_{A}\right)\right], \\
a_{56}=\frac{2 \mu}{r_{A}^{2}}\left[\left(-n+n^{2}\right) \mathrm{H}_{n}^{(2)}\left(k_{\beta} r_{A}\right)-\left(n k_{\beta} r_{A}\right) \mathrm{H}_{n+1}^{(2)}\left(k_{\beta} r_{A}\right)\right], \\
a_{57}=-\mathrm{i} k_{z} \frac{2 \mu}{r_{A}^{2}}\left[\left(-n+n^{2}-k_{\beta}^{2} r_{A}^{2}\right) \mathrm{H}_{n}^{(2)}\left(k_{\beta} r_{A}\right)+\left(k_{\beta} r_{A}\right) \mathrm{H}_{n+1}^{(2)}\left(k_{\beta} r_{A}\right)\right], \\
a_{58}=\lambda_{f 2}\left(k_{\alpha_{f 2}}^{2}+k_{z}^{2}\right) \mathrm{J}_{n}\left(k_{\alpha_{f 2}} r_{A}\right), \\
a_{61}=0, \\
a_{62}=\left[\left(n-n^{2}\right) \mathrm{J}_{n}\left(k_{\alpha} r_{A}\right)+\left(n k_{\alpha} r_{A}\right) \mathrm{J}_{n+1}\left(k_{\alpha} r_{A}\right)\right],
\end{gathered}
$$




$$
\begin{aligned}
& a_{63}=\left[\left(n-n^{2}+\frac{k_{\beta}^{2} r_{A}^{2}}{2}\right) \mathbf{J}_{n}\left(k_{\beta} r_{A}\right)+\left(-k_{\beta} r_{A}\right) \mathbf{J}_{n+1}\left(k_{\beta} r_{A}\right)\right], \\
& a_{64}=-n \mathrm{i} k_{z}\left[(-n+1) \mathrm{J}_{n}\left(k_{\beta} r_{A}\right)+\left(k_{\beta} r_{A}\right) \mathrm{J}_{n+1}\left(k_{\beta} r_{A}\right)\right], \\
& a_{65}=\left[\left(n-n^{2}\right) \mathrm{H}_{n}^{(2)}\left(k_{\alpha} r_{A}\right)+\left(n k_{\alpha} r_{A}\right) \mathrm{H}_{n+1}^{(2)}\left(k_{\alpha} r_{A}\right)\right], \\
& a_{66}=\left[\left(n-n^{2}+\frac{k_{\beta}^{2} r_{A}^{2}}{2}\right) \mathrm{H}_{n}^{(2)}\left(k_{\beta} r_{A}\right)+\left(-k_{\beta} r_{A}\right) \mathrm{H}_{n+1}^{(2)}\left(k_{\beta} r_{A}\right)\right] \text {, } \\
& a_{67}=-n \mathrm{i} k_{z}\left[(-n+1) \mathrm{H}_{n}^{(2)}\left(k_{\beta} r_{A}\right)+\left(k_{\beta} r_{A}\right) \mathrm{H}_{n+1}^{(2)}\left(k_{\beta} r_{A}\right)\right], \\
& a_{68}=0, \\
& a_{71}=0, \\
& a_{72}=-\mathrm{i} k_{z}\left[\left(n r_{A}\right) \mathrm{J}_{n}\left(k_{\alpha} r_{A}\right)-\left(k_{\alpha} r_{A}^{2}\right) \mathrm{J}_{n+1}\left(k_{\alpha} r_{A}\right)\right], \\
& a_{73}=\left[\frac{-\mathrm{i} k_{z}}{2} n r_{A} \mathrm{~J}_{n}\left(k_{\beta} r_{A}\right)\right] \text {, } \\
& a_{74}=\left\{\frac{1}{2 r_{A}}\left(k_{\beta}^{2} r_{A}^{2}-k_{z}^{2} r_{A}^{2}\right)\left[n \mathbf{J}_{n}\left(k_{\beta} r_{A}\right)-\left(k_{\beta} r_{A}\right) \mathbf{J}_{n+1}\left(k_{\beta} r_{A}\right)\right]\right\}, \\
& a_{75}=-\mathrm{i} k_{z}\left[\left(n r_{A}\right) \mathrm{H}_{n}^{(2)}\left(k_{\alpha} r_{A}\right)-\left(k_{\alpha} r_{A}^{2}\right) \mathrm{H}_{n+1}^{(2)}\left(k_{\alpha} r_{A}\right)\right], \\
& a_{76}=\left[\frac{-\mathrm{i} k_{z}}{2} n r_{A} \mathrm{H}_{n}^{(2)}\left(k_{\beta} r_{A}\right)\right] \\
& a_{77}=\left\{\frac{1}{2 r_{A}}\left(k_{\beta}^{2} r_{A}^{2}-k_{z}^{2} r_{A}^{2}\right)\left[n \mathrm{H}_{n}^{(2)}\left(k_{\beta} r_{A}\right)-\left(k_{\beta} r_{A}\right) \mathrm{H}_{n+1}^{(2)}\left(k_{\beta} r_{A}\right)\right]\right\} \text {, } \\
& a_{78}=0, \\
& a_{81}=0, \\
& a_{82}=\left[\frac{n}{r_{A}} \mathbf{J}_{n}\left(k_{\alpha} r_{A}\right)-k_{\alpha} \mathrm{J}_{n+1}\left(k_{\alpha} r_{A}\right)\right] \text {, } \\
& a_{83}=\left[\frac{n}{r_{A}} \mathbf{J}_{n}\left(k_{\beta} r_{A}\right)\right], \\
& a_{84}=-\mathrm{i} k_{z}\left[\frac{n}{r_{A}} \mathrm{~J}_{n}\left(k_{\beta} r_{A}\right)-k_{\beta} \mathrm{J}_{n+1}\left(k_{\beta} r_{A}\right)\right] \text {, } \\
& a_{85}=\left[\frac{n}{r_{A}} \mathrm{H}_{n}^{(2)}\left(k_{\alpha} r_{A}\right)-k_{\alpha} \mathrm{H}_{n+1}^{(2)}\left(k_{\alpha} r_{A}\right)\right] \text {, }
\end{aligned}
$$




$$
\begin{gathered}
a_{86}=\left[\frac{n}{r_{A}} \mathrm{H}_{n}^{(2)}\left(k_{\beta} r_{A}\right)\right], \\
a_{87}=-\mathrm{i} k_{z}\left[\frac{n}{r_{A}} \mathrm{H}_{n}^{(2)}\left(k_{\beta} r_{A}\right)-k_{\beta} \mathrm{H}_{n+1}^{(2)}\left(k_{\beta} r_{A}\right)\right], \\
a_{88}=-\left[\frac{n}{r_{A}} \mathrm{~J}_{n}\left(k_{\alpha_{f 2}} r_{A}\right)-k_{\alpha_{f 2}} \mathrm{~J}_{n+1}\left(k_{\alpha_{f 2}} r_{A}\right)\right] .
\end{gathered}
$$

If the source is placed in the outer fluid (Fluid 1), the terms $b_{j}$ are defined as:

$$
\begin{aligned}
b_{1}=\frac{\mathrm{i}}{2} \varepsilon_{n}(-1)^{n} \mathrm{H}_{n}^{(2)}\left(k_{\alpha_{f 1}} r_{0}\right) \lambda_{f 1} \frac{\omega^{2}}{\alpha_{f 1}^{2}} \mathrm{~J}_{n}\left(k_{\alpha_{f 1}} r_{B}\right), \\
b_{2}=0, \\
b_{3}=0, \\
b_{4}=-\frac{\mathrm{i}}{2} \varepsilon_{n}(-1)^{n} \mathrm{H}_{n}^{(2)}\left(k_{\alpha_{f 1}} r_{0}\right)\left[\frac{n}{r_{B}} \mathrm{~J}_{n}\left(k_{\alpha_{f 1}} r_{B}\right)-k_{\alpha_{f 1}} \mathrm{~J}_{n+1}\left(k_{\alpha_{f 1}} r_{B}\right)\right], \\
b_{5}=0, \\
b_{6}=0, \\
b_{7}=0, \\
b_{8}=0 .
\end{aligned}
$$

If the source is placed in the inner fluid (Fluid 2), the terms $b_{j}$ are defined as:

$$
\begin{aligned}
& b_{1}=0, \\
& b_{2}=0, \\
& b_{3}=0, \\
& b_{4}=0, \\
& b_{5}=\frac{\mathrm{i}}{2} \varepsilon_{n}(-1)^{n} \mathrm{~J}_{n}\left(k_{\alpha_{f 2}} r_{0}\right) \lambda_{f 2} \frac{\omega^{2}}{\alpha_{f 2}^{2}} \mathrm{H}_{n}^{(2)}\left(k_{\alpha_{f 2}} r_{A}\right), \\
& b_{6}=0, \\
& b_{7}=0, \\
& b_{8}=-\frac{1}{2} \varepsilon_{n}(-1)^{n} \mathbf{J}_{n}\left(k_{\alpha_{f 2}} r_{0}\right)\left[\frac{n}{r_{A}} \mathrm{H}_{n}^{(2)}\left(k_{\alpha_{f 2}} r_{A}\right)-k_{\alpha_{f 2}} \mathrm{H}_{n+1}^{(2)}\left(k_{\alpha_{f 2}} r_{A}\right)\right] .
\end{aligned}
$$




\section{References}

[1] G. Liu, J. Qu, Transient wave propagation in a circular annulus subjected to transient excitation on its outer surface, Journal of the Acoustical Society of America 104 (3) (1998) 1210-1220.

[2] J. Chung, J. Lee, Vibration analysis of a nearly axisymmetric shell structure using a new finite ring element, Journal of Sound and Vibration 219 (1) (1999) 35-50.

[3] N. Veksler, J. Izbicki, J. Conoir, Bending A wave in the scattering by a circular cylindrical shell: its relation with the bending free modes, Journal of the Acoustical Society of America 96 (1) (1994) 287-293.

[4] X.L. Bao, P.K. Raju, H. Uberall, Circumferential waves on an immersed, fluid-filled elastic cylindrical shell, Journal of the Acoustical Society of America 105 (5) (1999) 2704-2709.

[5] G. Maze, J. Cheeke, X. Li, Z. Wang, Coupled guided acoustic modes in water-filled thin-walled tubes, Journal of the Acoustical Society of America 110 (5) (2001) 2295-2300.

[6] A. Tadeu, L. Godinho, 3-D wave scattering by a fixed cylindrical inclusion submerged in a fluid medium, Engineering Analysis with Boundary Elements 23 (1999) 745-756.

[7] Y.H. Pao, C.C. Mow, Diffraction of Elastic Waves and Dynamic Stress Concentrations, Rand Corporation, Santa Monica, CA, 1973.

[8] A.L. Kurkjian, S.K. Chang, Acoustic multipole sources in fluid-filled boreholes, Geophysics 51 (1) (1986) 148-163.

[9] A. Tadeu, E. Kausel, Green's functions for two-and-a-half dimensional elastodynamic problems, Journal of Engineering Mechanics-American Society of Civil Engineers 126 (10) (2000) 1093-1097.

[10] M. Bouchon, K. Aki, Discrete wave-number representation of seismic-source wave field, Bulletin of the Seismological Society of America 67 (1977) 259-277.

[11] A. Tadeu, L. Godinho, P. Santos, Wave motion between two fluid filled boreholes in an elastic medium, EABEEngineering Analysis with Boundary Elements 26 (2) (2002) 101-117.

[12] L. Godinho, J. António, A. Tadeu, 3-D sound scattering by rigid barriers in the vicinity of tall buildings, Journal of Applied Acoustics 62 (11) (2001) 1229-1248.

[13] G.N. Watson, A Treatise on the Theory of Bessel Functions, 2nd Edition, Cambridge University Press, Cambridge, UK, 1980.

[14] R. Phinney, Theoretical calculation of the spectrum of first arrivals in layered elastic mediums, Journal of Geophysical Research 70 (1965) 5107-5123.

[15] E. Kausel, J. Roesset, Frequency domain analysis of undamped systems, Journal of Engineering MechanicsAmerican Society of Civil Engineers 118 (4) (1992) 721-734.

[16] K. Graff, Wave Motion in Elastic Solids, Dover Publication, Inc., New York, 1975. 